difference seen amongst the varying seniority of clinicians. These findings were corroborated by the sEMG readings.

Conclusions: ENT surgeons who perform prolonged microscopic work are at risk of musculoskeletal pain, which correlates with surgical experience suggesting an element of postural adaptation. Our prototype ergonomic support system can help delay the sensations of postural strain.

doi:10.1017/S002221511600270X

Free Papers (F712)

ID: 712.2

Endoscopic management of cholesteatoma with Khan's Endoholder

Presenting Author: Mubarak Khan

Mubarak Khan

Mimer medical college

Learning Objectives: Endoscopic ear surgery provides a minimally invasive approach to the middle ear. The disadvantage of endoscopic ear surgery is that it is a singlehanded surgical technique. The nondominant hand of the surgeon is utilized for holding and manipulating the endoscope. This necessitated the need for the development of an endoscope holder that would allow both hands to be free for surgical manipulation. The aim of this article is to report our preliminary experience using our newly designed and developed endoscope holder, which allowed us to perform cholesteatoma surgery utilizing both hands for surgery.

Study Design: Retrospective nonrandomized clinical study.

Methods: The endoscope holder was designed and developed to aid in endoscopic cholesteatoma surgery and to overcome the disadvantage of single-handed endoscopic surgery. The design of the endoscope holder is described in detail, along with instructions on how it can be used. A total of 87 endoscope holder-assisted cholesteatoma surgeries were performed to evaluate the feasibility of a two-handed technique and to evaluate the results of surgery.

Results: Out of 87 Endoholder assisted cholesteatoma surgeries, 82 surgeries were performed exclusively with Endoholder and 5 needed combined approach (endoscope + microscope) suggesting 94\% success in using exclusive Endoholder for endoscopic management of cholesteatoma.

The endoscope holder eliminates the disadvantages of single-handed surgery and is a good option for those who wish to perform endoscopic cholesteatoma surgery using both hands.

Conclusion: The study reports the successful application and use of the endoscope holder in a two-handed technique of endoscopic cholesteatoma management.
doi:10.1017/S0022215116002711

\section{Free Papers (F712)}

\section{ID: 712.3}

Long term hearing outcomes with the shape memory Nitinol stapes prosthesis: 10 year results

\section{Presenting Author: Rebecca Heywood}

Rebecca Heywood ${ }^{1}$, Mark Quick ${ }^{2}$, Marcus Atlas ${ }^{3}$

${ }^{1} \mathrm{Ng}$ Teng Fong General Hospital, ${ }^{2}$ Sir Charles

Gairdner Hospital, ${ }^{3}$ Ear Science Institute

Australia

\section{Learning objectives:}

1. Understand the variability that ensues during crimping of stapes prostheses

2. Understand the benefits conferred by self-crimping shape memory prostheses

3. Learn about long term stability of hearing outcomes using self-crimping shape memory prostheses

Introduction: Self-crimping stapes pistons were introduced to remove the manual component of the crimping process during stapedectomy with a view to producing stable long term hearing improvement in a reproducible manner and reducing trauma to the middle and inner ear. The objective of this study was to assess the long term clinical hearing outcomes and their stability following stapedectomy using a self-crimping shape memory Nitinol prosthesis over a 10 year period.

Methods: Retrospective case review was performed in a tertiary referral centre. Thirteen adult patients underwent fourteen stapedectomy procedures using a self-crimping shape memory Nitinol prosthesis between November 2003 and February 2005. Pure tone audiometry was performed preoperatively, at three monthly intervals up to two years and at five and ten years postoperatively.

Results: Mean postoperative air conduction $(0.5,1,2$ and $3 \mathrm{kHz}$ ) was $24.4 \mathrm{~dB}$ (standard deviation 8.3 ) at 1 year and $29.6 \mathrm{~dB}(11.2)$ at 10 years. Mean postoperative bone conduction $(0.5,1,2$ and $3 \mathrm{kHz})$ was $18.6(8.0)$ at 1 year and 25.0 (12.0) at 10 years. Mean postoperative air bone gap $(0.5,1$, 2 and $3 \mathrm{kHz}$ ) was $5.5 \mathrm{~dB}(3.0)$ at 1 year and $4.8 \mathrm{~dB}(3.9)$ at 10 years. Mean air bone gap closure was 23.3 (12.6) at 1 year and $24.2(9.9)$ at 10 years. Mean change in high tone bone conduction level $(1,2$ and $4 \mathrm{kHz})$ was $5.4 \mathrm{~dB}(6.0)$ at 1 year and $-0.2 \mathrm{~dB}(7.0)$ at 10 years, a mean deterioration of $5.6 \mathrm{~dB}(0.6 \mathrm{~dB}$ per year $)$.

Conclusions: Excellent closure of the air bone gap is demonstrated and it remains stable over at least ten years. There is no evidence that circumferential firm fixation of the prosthesis hook around the long process of incus has a detrimental effect in the long term. 